\title{
RESEARCH
}

Open Access

\section{Characteristics and progression of patients with frontotemporal dementia in a regional memory clinic network}

Mélanie Leroy ${ }^{1}$, Maxime Bertoux', Emilie Skrobala², Elisa Mode ${ }^{3}$, Catherine Adnet-Bonte ${ }^{1}$, Isabelle Le Ber ${ }^{4,5}$, Stéphanie Bombois ${ }^{1}$, Pascaline Cassagnaud ${ }^{1}$, Yaohua Chen ${ }^{1}$, Vincent Deramecourt ${ }^{1}$, Florence Lebert ${ }^{1}$, Marie Anne Mackowiak ${ }^{1}$, Adeline Rollin Sillaire ${ }^{1}$, Marielle Wathelet ${ }^{6}$, Florence Pasquier ${ }^{1}$, Thibaud Lebouvier ${ }^{1 *}$ (D) and the Méotis network

\begin{abstract}
Background: Due to heterogeneous clinical presentation, difficult differential diagnosis with Alzheimer's disease (AD) and psychiatric disorders, and evolving clinical criteria, the epidemiology and natural history of frontotemporal lobar degeneration (FTD) remain elusive. In order to better characterize FTD patients, we relied on the database of a regional memory clinic network with standardized diagnostic procedures and chose AD patients as a comparator.

Methods: Patients that were first referred to our network between January 2010 and December 2016 and whose last clinical diagnosis was degenerative or vascular dementia were included. Comparisons were conducted between FTD and AD as well as between the different FTD syndromes, divided into language variants (IVFTD), behavioral variant (bvFTD), and FTD with primarily motor symptoms (mFTD). Cognitive progression was estimated with the yearly decline in Mini Mental State Examination (MMSE).
\end{abstract}

Results: Among the patients that were referred to our network in the 6-year time span, 690 were ultimately diagnosed with FTD and 18,831 with AD. Patients with FTD syndromes represented $2.6 \%$ of all-cause dementias. The age-standardized incidence was 2.90 per 100,000 person-year and incidence peaked between 75 and 79 years. Compared to AD, patients with FTD syndromes had a longer referral delay and delay to diagnosis. Patients with FTD syndromes had a higher MMSE score than AD at first referral while their progression was similar. mFTD patients had the shortest survival while survival in bvFTD, IVFTD, and AD did not significantly differ. FTD patients, especially those with the behavioral variant, received more antidepressants, anxiolytics, and antipsychotics than AD patients.

Conclusions: FTD syndromes differ with AD in characteristics at baseline, progression rate, and treatment. Despite a broad use of the new diagnostic criteria in an organized memory clinic network, FTD syndromes are longer to diagnose and account for a low proportion of dementia cases, suggesting persistent underdiagnosis. Congruent with recent publications, the late peak of incidence warns against considering FTD as being exclusively a youngonset dementia.

Keywords: Frontotemporal dementia, Epidemiology, Progression, Dementia

\footnotetext{
* Correspondence: thibaud.lebouvier@chru-lille.fr

'Univ. Lille, Inserm, CHU Lille, Lille Neuroscience \& Cognition, CNRMAJ,

LiCEND, DistAlz, F-59000 Lille, France

Full list of author information is available at the end of the article
}

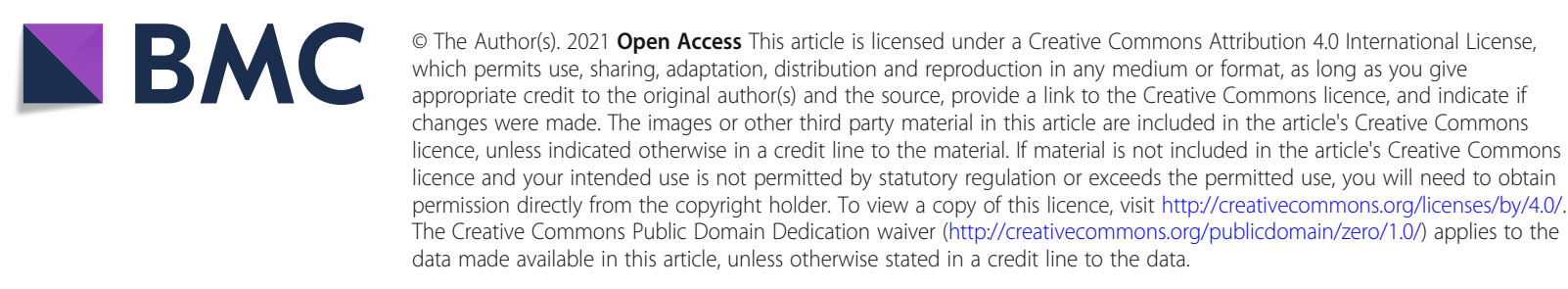




\section{Background}

Frontotemporal lobar degeneration (FTD) is the second leading cause of early-onset dementia after Alzheimer's disease (AD) [1]. FTD is characterized by changes in behavior and/or language due to the relatively selective atrophy of the frontal and temporal lobes [2]. In the past decade, the nosology of FTD has evolved outstandingly, prompting changes in diagnostic criteria. There are three main clinical presentations of FTD. The behavioral variant of FTD (bvFTD) is defined by an early and prominent behavioral and dysexecutive syndrome, whose core symptoms were revised by Rascovsky et al. in 2011 [3]. The two language variants of FTD (lvFTD) include the semantic and non-fluent presentations of primary progressive aphasia (PPA), also defined by updated clinical criteria [4]. In addition, FTD can initially present with motor symptoms (mFTD) such as features of atypical parkinsonism (progressive supranuclear palsy [PSP] and corticobasal syndrome [CBS]) [5].

Although being an umbrella term underlain by $>20$ different possible pathologies [6], FTD stands as a unifying entity because of the lack of correlations between FTD syndromes and pathology [7]. bvFTD, for example, can be underlain by tau, TDP-43 or rarer pathologies, and on the contrary, one single pathology, such as PSP, can manifest with several clinical syndromes [6]. One exception to the unpredictability of the underlying pathology is the identification of a causal genetic mutation. Patients with FTD syndromes have a positive family history in $26-31 \%$ [8], highlighting the importance of genetics. The most common FTD mutations, all linked to a specific pathology, are found on MAPT, PGRN, and C9ORF72 genes [8].

FTD prevalence was estimated between $0.01-4.61$ per 1000 person and the incidence between $0.01-2.5$ per 1000 person/year [9]. In recent dementia cohorts, FTD cases have been found to account for 1.6 to $7 \%$ of dementia cases $[10,11]$. However, those figures need to be considered with caution. First, FTD is still underdiagnosed: neuropathological studies performed in communities where brain donation reaches a high level of acceptance show that as much as $5-9 \%$ of the elderly population with or without cognitive impairment at death has FTD pathology $[12,13]$. It has been previously estimated that about $40 \%$ of FTD are misdiagnosed [14] and time to diagnosis is longer than for other dementias $[15,16]$. Second, with some exceptions [17], most past estimations have been done using the previous Lund and Manchester [18] or Neary criteria [19]. Yet, the revised clinical criteria and the addition of new syndromes to the FTD spectrum outdate previous publications. Third, advances in neuropsychology, neuroimaging, and cerebrospinal fluid (CSF) biomarkers and genetics have improved FTD diagnosis in challenging situations such as psychiatric, amnestic, or late-onset presentations of the disease [20-22].

However, beyond research purposes, whether improving FTD diagnosis at the population level would stand a cost-benefit analysis is a subject that should be addressed open-mindedly. Indeed, one could argue that differential dementia diagnosis workup is a costly venture [23] that can be questioned in the absence of disease-modifying treatments. The demonstration that FTD diagnosis is associated with different prognoses and therapeutic approaches in routine care would advocate against a symptomatic approach of dementia.

Thus, data sharing on current FTD diagnoses and management is needed. We undertook the present study in a large regional memory clinic (MC) network to get a better overview of the incidence, characteristics and natural history of FTD syndromes defined using recent diagnostic criteria. The objectives were to study the characteristics of the FTD patients referred to the network from January 2010 to December 2016, including age at onset, time to diagnosis, clinical presentations, cognitive progression, and treatment.

\section{Patients and methods \\ Patient selection}

Founded in 1993, the Méotis network is the first French $\mathrm{MC}$ network, involving $30 \mathrm{MCs}$ in the French Nord and Pas-de-Calais departments, sharing data within a common patient database since 1997. Meotis database reached a caseload of over 104,000 patients in 2018, representing more than 350,000 visits [24]. In all MCs, a multidisciplinary assessment is performed by neurologists, geriatricians, psychologists, dedicated nurses, and social workers; whenever necessary, patients can be assessed by psychiatrists, speech therapists, and dedicated nurses. Diagnostic work-up is harmonized throughout the network, and standardized data on patient characteristics and healthcare activity are systematically collected. All harmonized data are monitored and computerized by a data manager in the tertiary-referral Memory Resources and Research Center (MRRC) of the Lille University Hospital.

We included patients that were referred for the first time to one of the network's MC from January 2010 to December 2016 and whose last clinical diagnosis during the follow-up was FTD, AD, or other causes of dementia. We first extracted all dementia cases to calculate the respective proportions of $\mathrm{AD}$ and FTD syndromes. Then, we focused on the subpopulation of AD and FTD syndromes for systematic comparisons. Since AD is the dominant cause of dementia, AD patients were chosen as a comparator. Data extraction was performed on September 2019, 33 months after the end of the inclusion 
period. For the few patients that received a diagnosis of bvFTD and lvFTD before the new criteria were published and were not followed up beyond 2011, we checked retrospectively that they fulfilled the revised diagnostic criteria. The bvFTD group comprised pure bvFTD [3] and a minority of patients with associated amyotrophic lateral sclerosis. The lvFTD group included a semantic and non-fluent agrammatic PPA [4] as well as rarer PPA variant such as apraxia of speech. The mFTD group comprised the PSP [25] and CBS [26] patients. Patients with overt motor neurone disease at presentation are usually not referred to our network because of a specialized regional amyotrophic lateral sclerosis care pathway.

\section{Data collection}

We extracted the following data from the Méotis database: sex, age at first referral, referral delay, age at diagnosis, symptom onset, and diagnostic procedures. We collected the Mini Mental State Examination (MMSE) [27] and the short 4-item Instrumental Activities of Daily Living (IADL-4) [28] scores at first referral. In this article, IADLs score was calculated by summing up the number of maintained activities (ranging from 0 (full dependence) to 4 (complete autonomy)). The referral delay was defined as the interval, expressed in months, between symptoms onset (declared by the patient and caregiver) and first referral to the network. The clinical follow-up was defined as the interval, expressed in years, between the first and the last visit within the network. The survival was defined as the interval, expressed in years, between disease onset and death. Drug treatment was recorded at every visit. A patient was considered under a specific drug treatment if it was recorded at least once during follow-up.

Only the last clinical diagnosis was considered in this study because of its higher accuracy. The last diagnosis was the one made or kept after all diagnostic procedures and retained at follow-up. Diagnosis wandering was defined as the time from first referral to the last retained clinical diagnosis.

The date of death was retrieved from the National Institute of Statistics and Economic Studies (French: Institut national de la statistique et des études économiques) national death database thanks to the MatchID tool (https://deces.matchid.io/) on September 2020.

The datasets considered in the current study are available from the corresponding author on reasonable request. The database was declared to the ad hoc commission (Commission Nationale Informatique et Libertés (CNIL)) protecting personal data (\#2146189 V1). Privacy and confidentiality rules were respected.

\section{Statistical analysis}

Quantitative variables were described by the mean and standard deviation if the distribution was normal or by the median and interquartile range otherwise. Qualitative variables were described by the numbers and percentages of each modality.

Diagnostic subtypes (bvFTD, FTD mFTD and AD) were described and compared across all parameters. Quantitative variables were analyzed by an ANOVA or the Kruskal-Wallis non-parametric equivalent. Qualitative variables were compared by an exact chi-square or Fisher's test (in the case of a theoretical number of cases below 5). A Bonferroni correction was applied to posthoc comparisons of the FTD subgroups with respect to the AD group. The effect size was calculated as the standardized mean difference (for quantitative variables) and the Cramer's V coefficient (for qualitative variables). A mixed linear model analyzed the evolution of the MMSE over time. The factors introduced into the model were time, diagnosis, and the interaction between diagnosis and time.

Incidence rates were calculated as the number of incident cases divided by the total number of person-years (py) for the catchment area over the 7 years. All rates were calculated using the reference population of the corresponding geographic area estimated by the French National Institute of Demographic Research (INED) on January 2015, as population at risk. Therefore, no variation was assumed during the 7 years of the study period. Age-standardized rates were calculated using the Revised European Standard Population 2013 (ESP2013). Results were presented in cases per 100,000 personyears.

Concerning mortality, median survival time after diagnosis was calculated for each diagnostic subtype, survival was estimated using the Kaplan-Meier model, and the log-rank test was used to test of differences in survival curves according to diagnostic subtype. Hazard ratios (HRs) were also adjusted for age and sex using Cox regression.

The analyses were performed using SAS software (version 9.4).

\section{Results \\ Study population}

Data from 26,525 demented patients followed in the network and fulfilling inclusion criteria were extracted. Among them, 2369 have first been seen at the MRRC (Lille tertiary-referral MC) and 24,156 first at one of the MCs belonging to the network.

During the 7 years of follow-up, 690 incident cases of FTD syndromes were identified, giving a crude incidence rate of 2.42 per 100,000 person-years (Table 1). The FTD incidence across age groups at diagnosis reached 
Table 1 FTD incidence rates, number of cases, and number of person-years by age group

\begin{tabular}{llll}
\hline Age group at diagnosis & Number of cases & Number of person-years & Incidence (per 100,000 person-years) \\
\hline $0-39$ & 2 & $14,854,742$ & 0.01 \\
$40-44$ & 3 & $1,922,550$ & 0.16 \\
$45-49$ & 8 & $1,842,470$ & 0.43 \\
$50-54$ & 25 & $1,862,287$ & 1.34 \\
$55-59$ & 61 & $1,796,207$ & 3.40 \\
$60-64$ & 95 & $1,703,044$ & 5.58 \\
$65-69$ & 123 & $1,490,146$ & 8.25 \\
$70-74$ & 110 & 828,709 & 13.27 \\
$75-79$ & 126 & 842,548 & 14.95 \\
$80-84$ & 92 & 725,613 & 12.68 \\
$\geq 85$ & 45 & 676,866 & 6.65 \\
Total & 690 & $28,545,202$ & 2.42 \\
\hline
\end{tabular}

its peak in the 75-to-79 year-old group, with an incidence rate of 14.95 per 100,000 person-years. The agestandardized incidence rate was 2.90 per 100,000 person-years.

FTD syndromes represented $2.6 \%$ of the studied population, as compared with $71 \%$ AD (Fig. 1a). Among FTD syndromes, $64 \%$ were bvFTD, $17 \%$ lvFTD, and $18 \%$ mFTD (Table 2). The proportion of FTD syndromes was higher in the MRRC (8.1\%) than elsewhere (2.0\%).

\section{Characteristics of patients with FTD syndromes}

The sex ratio significantly differed between AD and FTD patients $(p<0.0001, d=0.1)$ (Table 2). Men represented $47 \%$ of FTD and only $30 \%$ of AD patients. Patients with FTD syndromes were younger than those with $\mathrm{AD}$ at first referral (70.4 vs. 80.6 years, $p<0.0001$ ), and bvFTD patients were younger than the remaining FTD syndromes (69.4 vs. 72.3 years).

MMSE scores at first referral were higher in FTD syndromes than in $\mathrm{AD}$ (21.8 vs. 18.9, $p<0.0001, d=0.5$ ). Likewise, the median IADL-4 score was higher in patients with FTD syndromes compared to AD patients (3 vs. $2, p<0.0001, d=0.5)$, favoring a more preserved autonomy in instrumental activities.

Among the FTD syndromes, a positive family history of dementia was identified in $14 \%$, as compared with $2.3 \%$ of AD patients $(p<0.0001, d=0.1)$. Among the 294 FTD syndromes referred to the MRRC, a genetic mutation was detected in $34 \%$ of the 99 patients in whom the genetic analysis was performed (47\% in C9Orf72, 32\% in PGRN, and $21 \%$ in MAPT genes). Mutations were more likely to be retrieved in bvFTD (95\%) than in lvFTD (5\%) or mFTD (0\%). See Table 1 for detailed comparisons between FTD syndromes and AD.

\section{Diagnosis of FTD syndromes}

We then systematically studied the time to referral, time to diagnosis and diagnostic workup of FTD compared to AD patients. Referral delay was longer for FTD syndromes compared to $\mathrm{AD}$ (37.6 vs. 31.8 months, $p<$ $0.0001, d=0.4)$. Among the FTD syndromes, referral delay was the highest for bvFTD (40.0 vs. 33.3 months in other FTD). Diagnosis wandering was longer for FTD syndromes compared to $\mathrm{AD}$ (9.8 vs. 5.8 months, $p<$ $0.001, d=0.1$ ), but similar across FTD syndromes.

As part of the standardized dementia diagnosis procedure, all of our patients performed an MRI, if not contraindicated. The diagnostic workup of FTD patients in the whole Méotis network included more often a FDGPET and a lumbar puncture that the one of AD patients (23.2\% vs. $2.6 \%$ and $27.5 \%$ vs. $3.96 \%$ respectively, $p<$ 0.001 and $d=0.2$ for both comparisons, Table 2). Brain imaging and lumbar puncture were more consistently used in Lille MMRC both for AD and FTD diagnosis (our unshown data).

Correlations between clinical diagnoses and pathology were excellent in the 15 patients of the study population who came to autopsy. Among the patients with available pathological examination, the 4 in the bvFTD group had FTLD-TDP $(n=3)$ or FTLD-FUS $(n=1)$ pathologies. All 5 patients in the mFTD group had PSP or CBD pathology. All 6 patients in the AD group had AD pathology + / - cerebral amyloid angiopathy or Lewy body pathology ( $n=3$ and 2 , respectively).

\section{Natural history of FTD syndromes}

Cognitive progression estimated by the rate of MMSE decline was then assessed. Overall, there was no significant difference in the rate of MMSE decline between FTD syndromes and AD. Across FTD syndromes, 


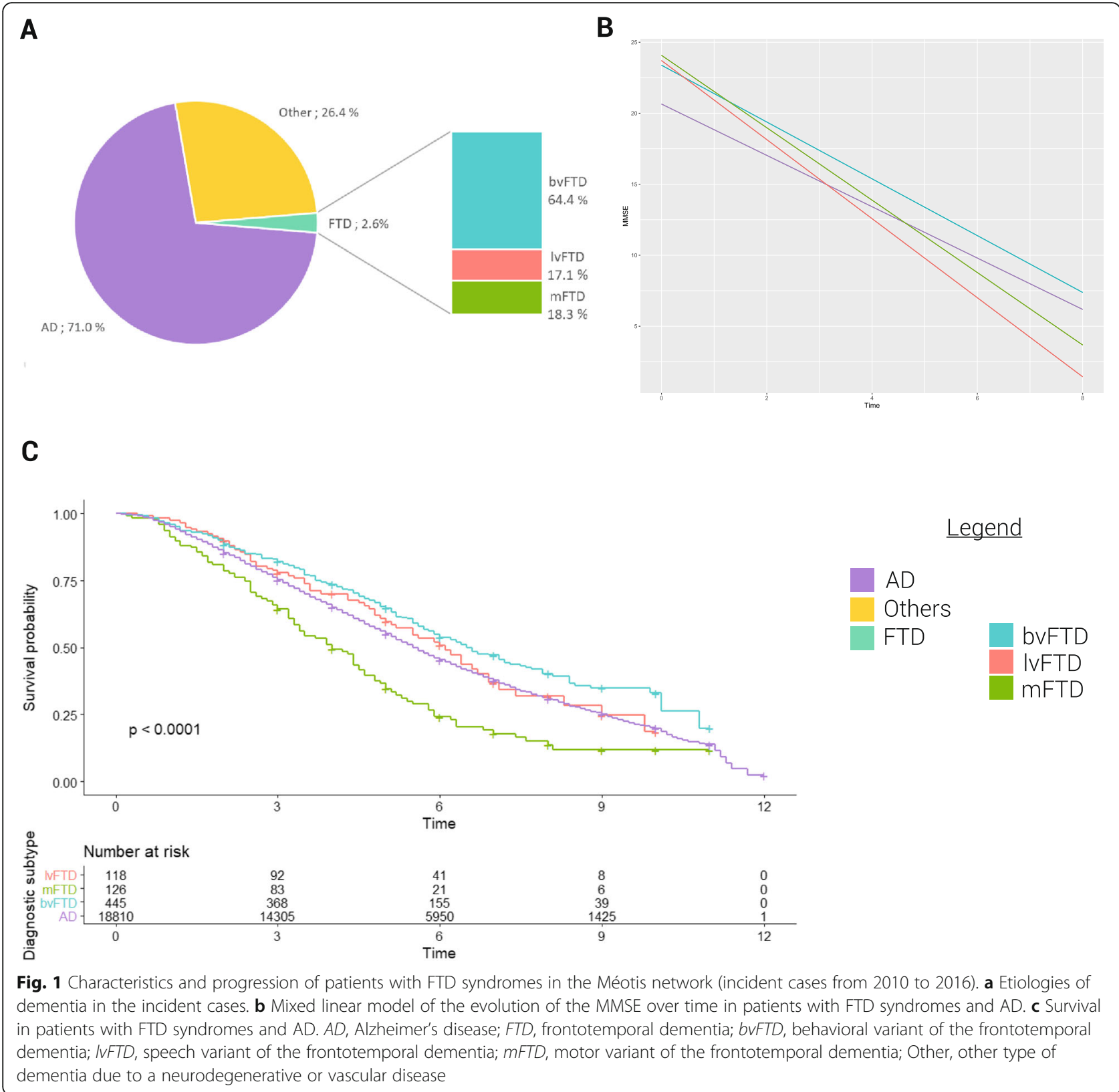

bvFTD did not significantly differ from AD in the rate of MMSE decline per year (the slope was $a_{\mathrm{bvFTD}}=-2.0$ in bvFTD against $a_{\mathrm{AD}}=-1.8$ in $\left.\mathrm{AD}, p=0.4\right)$. However, the decline was higher in lvFTD $\left(a_{\mathrm{lvFTD}}=-2.8\right)$ and $\mathrm{mFTD}$ $\left(a_{\mathrm{mFTD}}=-2.6\right)$ than in $\mathrm{AD}$ patients $(p=0.003$ and $p=$ 0.02 , respectively) (Fig. 1b). Follow-up was longer for FTD syndromes compared to AD (24.1 vs. 17.5 months, $p<0.0001, d=0.2$ ), and more specifically, bvFTD and lvFTD patients had a significantly longer follow-up than AD patients.

As of September 2020, 48\% of bvFTD, 53\% of lvFTD, $76 \%$ of $\mathrm{mFTD}$, and $59.1 \%$ of AD patients had died (Table 2).
The median survival time after diagnostic was 5.5 years for the entire sample and varied significantly according to the diagnosis subtype (6.5 years for bvFTD, 6.1 for lvFTD, 5.5 for AD, and 4.0 for mFTD, $p<0.001$ ) (Fig. 1c). Age (HR [CI $95 \%]=1.05[1.05-1.06]$ for 1 year, $p<0.001)$ and male sex (1.73 [1.67-1.80], $p<0.001)$ were significantly associated with an increased risk of death. After adjustment for age and sex, MFTD were significantly associated with a lower median survival as compared to AD (2.32 [1.89-2.84], $p<$ $0.001)$. There were no significant differences between $A D$ and bvFTD (1.10 [0.96-1.26], $p=0.179)$ and between AD and lvFTD (1.21 [0.94-1.55], $p=0.137)$. 
Table 2 Demographics and clinical features of FTD syndromes

\begin{tabular}{|c|c|c|c|c|c|c|c|c|c|c|c|}
\hline \multirow[b]{3}{*}{ Variable } & \multirow{2}{*}{\multicolumn{3}{|c|}{$\frac{\text { bvFTD }}{N=446}$}} & \multirow{2}{*}{\multicolumn{3}{|c|}{$\begin{array}{l}\text { IvFTD } \\
N=118\end{array}$}} & \multirow{2}{*}{\multicolumn{3}{|c|}{$\begin{array}{l}\mathrm{mFTD} \\
N=126\end{array}$}} & \multirow{2}{*}{\multicolumn{2}{|c|}{$\frac{A D}{N=18,831}$}} \\
\hline & & & & & & & & & & & \\
\hline & Value & NA & $p^{*}$ & Value & NA & $p^{*}$ & Value & NA & $p^{*}$ & Value & NA \\
\hline Women, $n(\%)$ & $232(52.2)$ & 0 & $<0.001$ & $68(57.6)$ & 57.6 & 0.01 & $68(54)$ & 0 & $<0.001$ & $13,162(69.9)$ & 0 \\
\hline Age at first visit, m (sd) & $69.4(10.3)$ & 0 & $<0.001$ & $72.7(9.5)$ & 0 & $<0.001$ & $72.0(8.0)$ & 0 & $<0.001$ & $80.6(7.5)$ & 0 \\
\hline Age at diagnosis, m (sd) & $70.2(10.3)$ & 0 & $<0.001$ & $73.6(9.7)$ & 0 & $<0.001$ & $72.8(8.1)$ & 0 & $<0.001$ & $81.0(7.3)$ & 0 \\
\hline Delay referral (months), m (sd) & $40.0(41.2)$ & 87 & $<0.001$ & $30.8(20.5)$ & 19 & 0.84 & $35.8(29.9)$ & 24 & 0.13 & $31.8(32.0)$ & 5370 \\
\hline Diagnosis wandering (months), m (sd) & $9.9(16.8)$ & 0 & $<0.001$ & $10.5(16.4)$ & 0 & $<0.001$ & $9.1(15.1)$ & 0 & $<0.001$ & $5.8(14.2)$ & 0 \\
\hline Clinical follow-up (months), m (sd) & $25.2(24.0)$ & 0 & $<0.001$ & $24.2(22.0)$ & 0 & $<0.001$ & $20.2(19.6)$ & 0 & 0,05 & $17.5(21.4)$ & 0 \\
\hline Dementia family history, $n(\%)$ & $75(16.8)$ & 0 & $<0.001$ & $11(9.3)$ & 0 & $<0.001$ & $13(10.3)$ & 0 & $<0.001$ & $508(2.7)$ & 0 \\
\hline MMSE at first visit, $m$ (sd) & $22.1(6.1)$ & 117 & 0.02 & $20.6(7.5)$ & 27 & 0.002 & $21.9(6.1)$ & 38 & $<0.001$ & $18.9(6.0)$ & 2246 \\
\hline IADL-4, median & 3 & 199 & $<0.001$ & 4 & 38 & $<0.001$ & 3 & 54 & $<0.001$ & 2 & 6689 \\
\hline PET in the network, $n(\%)$ & $117(26.2)$ & 0 & $<0.001$ & $18(15.2)$ & 0 & $<0,001$ & $25(19.8)$ & 0 & $<0.001$ & $484(2.5)$ & 0 \\
\hline CSF in the network, $n(\%)$ & $135(30.3)$ & 0 & $<0.001$ & $22(18.6)$ & 0 & $<0,001$ & $33(26.2)$ & 0 & $<0.001$ & $759(3.9)$ & 0 \\
\hline Death, $n(\%)$ & $213(48.4)$ & 0 & & $63(53.4)$ & 0 & & $96(76.2)$ & 0 & & $11,220(59.1)$ & 0 \\
\hline Survival in years, median & 6.5 & 0 & 0.18 & 6.1 & 0 & 0.14 & 4.0 & 0 & $<0.001$ & 5.5 & 0 \\
\hline Autopsy verification, $n(\%)$ & $4(0.8)$ & 0 & & $0(0)$ & 0 & & $5(4.0)$ & 0 & & $6(0.03)$ & 0 \\
\hline
\end{tabular}

$A D$ Alzheimer's disease, FTD frontotemporal dementia, bvFTD behavioral variant of the frontotemporal dementia, IADL Instrumental Activities of Daily Livings, IVFTD speech variant of the frontotemporal dementia, MFTD motor variant of the frontotemporal dementia, IADL-4 Instrumental Activities of Daily Living-4, MMSE Mini Mental State Examination, $m$ ( $s d$ ) mean (standard deviation), PET position emission tomography, CSF cerebrospinal Fluid. *Comparison to AD

\section{Treatment of FTD syndromes}

There were sharp differences in the therapeutic approach between FTD syndromes and AD. FTD patients received less anticholinesterase inhibitors (AChEI) and memantine than AD patients $(12.0 \%$ vs. $42.2 \%, p<$ $0.0001, d=0.1$ and $5.7 \%$ vs. $21.8 \%, p<0.0001, d=0.1$, respectively). Conversely, FTD patients received more antidepressants $(48.0 \%$ vs. $27.0 \%, p<0.0001)$, anxiolytics (33.2\% vs. $23.6 \%, p<0.0001, d=0.04)$, and antipsychotics $(17.5 \%$ vs. $13.1 \%, p=0.003, d=0.1)$ than $\mathrm{AD}$ patients. The difference between AD and FTD stemmed mostly from the bvFTD group where antidepressants (55.2\%), anxiolytics (38.3\%), and antipsychotics (24.4\%) were used the most $(p<0.0001$ and $d=0.1$ for the three comparison to AD). There was no significant difference in the use of hypnotics between groups (Fig. 2).

\section{Discussion}

The main findings of the present study are threefold: (1) despite new sets of criteria, diagnoses of FTD syndromes remained low in routine care in our regional memory clinic network; when diagnosed, bvFTD patients had longer referral delay and diagnostic wandering than AD patients; (2) the peak of incidence of bvFTD occurred between 75 and 79 years, clearly advocating against the conception of FTD as exclusively an early-onset dementia; (3) FTD syndromes differed from AD with regard to cognition and autonomy at baseline, cognitive decline, and disease duration; and (4) therapeutic strategies radically differed from the ones in AD.

\section{Misconceptions about FTD lead to underdiagnosis}

In this retrospective study, we calculated an FTD agestandardized incidence rate of $2.9 / 100.000$ py in our region. Our results stand in-between the ones of two recent studies using updated FTD criteria that found an incidence of $1.6 / 100.000$ py in the UK (Norfolk and Cambridgeshire counties) and 3.05/100.000 py in Italy (Leccia and Brescia provinces) $[17,29]$. However, while we used the same European reference population as our British colleagues, Logroscino et al. used the Italian population for standardization. Standardization of their incidence rate with the same European population yields an FTD agestandardized incidence rate of 2.78/100.000 py, strikingly similar to ours (our unshown data).

We found that FTD syndromes represented 3\% of the Méotis network caseload. Similar MC surveys in Netherlands [11] and Sweden [10] had 7\% and 3.6\% of FTD syndromes, respectively. However, all patients in the Dutch cohort were followed in the Alzheimer center of the VU University Medical Center (VUmc), a tertiary center where atypical dementias are likely to be addressed, possibly leading to an overrepresentation of FTD patients. Likewise, there was a $8.1 \%$ proportion of FTD patients in Lille tertiary center. A recent review on the epidemiology of FTD highlighted three studies with high methodological standards [9]. In these publications using the Lund and Manchester [18] or Neary [19] criteria, FTD syndromes accounted for 1.1\% [30], 3\% [31], and $3.8 \%$ [32] of dementia cases, which is consistent with our findings. 


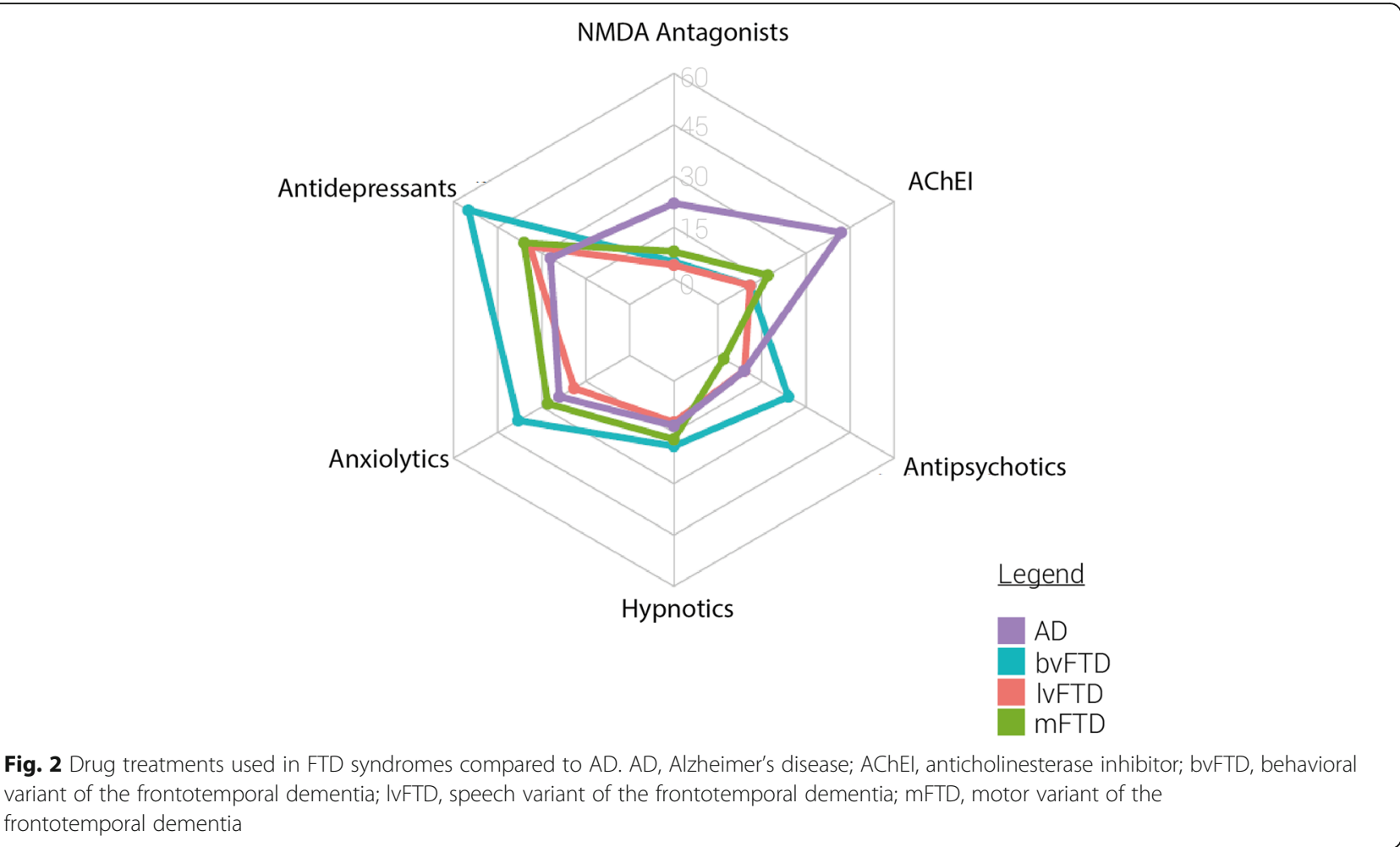

In sharp contrast, consistent with the underdiagnosis of FTD, systematic neuropathology surveys show much higher figures. In UK brain banks from donors (of whom two thirds had dementia), FTD represented $5.1 \%$ of diagnoses [33] and up to $9.4 \%$ of elderly people participating in a community brain donation program were found to have some FTD lesions at autopsy [13].

The reasons for FTD underdiagnosis are manifold. First, late-onset FTD are often overlooked. FTD is historically considered as a major cause of early onset dementia [1], which probably contributes to FTD diagnosis being overlooked in late-onset dementia. Yet, in recent studies with pathological confirmation, one fourth of FTD cases had an age at onset $>65$ years [34]. In the recent literature, there is a trend toward an increase in the age at diagnosis of FTD syndromes, which may relate to the increasing age at dementia diagnosis in recent surveys [24]. While older studies showed an age at diagnosis of 65.9 years [35], we found an age at diagnosis of 71.3 years, which compares to recent publications showing a mean age at diagnosis of 69.4 [36], 70.0 [37], or 71.3 years [29]. Interestingly, the peak of incidence occurred between 75 and 79 years in our survey as in the aforementioned Italian and English studies [17, 29], reminding that FTD is not only a dementia of early onset.

Second, the positive diagnosis of bvFTD and its differentiation with primary psychiatric disorders is another diagnostic challenge [38] that is reflected by the increased time to presentation and time to diagnosis of the bvFTD variants as compared with the others [39, 40]. Prolonged diagnostic wandering in bvFTD, associated in our study with an increased reliance on diagnostic biomarkers, seems to be a universal finding [15-17] and suggests that many cases could remain misdiagnosed. Future studies should focus on the exact determinants of the delay in referral and in diagnosis. Third, all the possible clinical presentations of FTD have not been thoroughly described and some are not taken into account by the available clinical criteria. The amnestic variant of FTD, in particular, is difficult to differentiate from $\mathrm{AD}[20,41]$ in particular in late-onset dementia [42]. Another example is the right temporal variant of FTD-although a recent publication proposing clinical criteria will contribute to fill the gap [43].

Overall, our survey confirms that FTD are still probably overlooked despite the use of novel clinical criteria and incorporation of new phenotypes. While progress has been made in the recognition of late-onset forms, differential diagnosis between FTD and AD remains a challenge, particularly in the oldest old, and bvFTD cases are probably still mistaken for primary psychiatric disorders.

\section{FTD syndromes differ with AD in baseline characteristics and natural history}

We found several key differences between FTD syndromes and $\mathrm{AD}$ at baseline. First, as we had previously 
shown [39], we confirmed that the MMSE score is higher in FTD. However, behavior, social cognition, and executive functions, the main domain impaired in bvFTD, are not properly assessed by the MMSE, which somewhat undermines the assumption that the general cognitive status is better preserved in FTD syndromes. The higher IADL-4 score in FTD compared to AD contrasted with past studies that retrieved either lower [44] or equal [45] autonomy. However, IADL-4 only assesses restriction in four activities (telephone, transportation, drug treatment, and finances) that are best associated with future dementia risk [28], thus preventing a direct comparison of our results with studies that employed the full ADL. The younger age and the better preservation of memory and visuo-motor functions may explain the lesser impairment found in FTD as compared to AD. Impaired functional capacity in bvFTD is primarily due to behavioral symptoms and impaired social cognition, and the routine (although complex) instrumental activities of the IADL-4 may not be the most representative of the loss of autonomy in FTD syndromes. Among the FTD syndromes, the lvFTD patients had the most preserved autonomy, as found in previous studies [44, 45].

Although FTD syndromes as a whole had a similar rate of MMSE decline to $\mathrm{AD}$, IVFTD and MFTD variants specifically showed a higher rate of MMSE decline in time. Additionally, lvFTD had a slightly lower score at baseline than the other variants. Since the MMSE relies mostly on language, aphasia has likely impacted the score in lvFTD. In recent studies, patterns of longitudinal MMSE decline across the FTD phenotypes have already been studied, and semantic dementia cases were shown to decline the most [46]. Regarding survival, we, as others [17], reported that $\mathrm{mFTD}$ had the more severe prognosis of FTD syndromes [17], followed by lvFTD and bvFTD. Despite similar MMSE decline rates between bvFTD and AD, mFTD patients had a significantly lower survival median.

\section{Therapeutic strategies in FTD}

The drug treatments used in FTD syndromes markedly differed from the ones used in $\mathrm{AD}$. These observations should be interpreted with caution since differences may only reflect different customs, and not different responses to treatment. However, clinical guidance on the symptomatic treatment of FTD is limited [47], prompting physicians to use psychotropic drugs that may be used nonspecifically in dementia, based on the medical needs and immediate efficacy. Hence, the prescription habits in FTD may also reflect the neuropsychiatric symptoms and treatment response of FTD patients.

A publication from the Boxer's group showed that offlabel use of AChEI and memantine in FTD was common in the USA in 2010 [48]. In our region and in the 2010-
2019 time span, we found that AChEI and memantine were used in only $12.0 \%$ and $5.7 \%$ of FTD syndromes, in accordance with recent data supporting lack of efficacy-or even deleterious effects in bvFTD and mFTD $([49,50]$, reviewed in $[51,52])$. The remaining prescriptions may reflect diagnostic hesitations with $\mathrm{AD}$ at the beginning of follow-up.

Antipsychotics and anxiolytics were more frequently used in FTD syndromes than in $\mathrm{AD}$, and the difference with $\mathrm{AD}$ was driven by the bvFTD variant. Antipsychotics are prescribed to treat agitation in dementia whatever the etiology (AD, FTD, or others) [53], although their use is restricted to patients with severe symptoms (aggression, agitation, or psychosis) who fail to respond adequately to other pharmacological and nonpharmacological treatments. The use of anxiolytics and antipsychotics in $38.3 \%$ and $24.4 \%$ of bvFTD patients, as opposed to $23.6 \%$ and $9.3 \%$ in $\mathrm{AD}$, is thus a reflection of the higher rate of productive behavioral symptoms (e.g., agitation, aggression, and psychosis) in this variant. However, the low rate of antipsychotics use in FTD demonstrated that physicians took into account the alerts on side effects $[54,55]$ and increased mortality rate [56] in FTD and dementia patients treated with antipsychotics. The black box warning from the Food and Drug Administration was followed by a similar warning from the French Haute Autorité de Santé in 2009 (https://www.has-sante.fr/ jcms/c_885227/fr/limiter-la-prescription-de-neuroleptiques -dans-la-maladie-d-alzheimer) that had found a strong echo in the neurologic and geriatric communities.

The most remarkable difference however regarded the prescription of antidepressants, which was twice as important in bvFTD (55.2\%) as in AD (27.0\%). Indeed, although results are mixed, comprehensive reviews of the evidence from clinical trials favored the use of selective serotonin reuptake inhibitors to treat behavioral symptoms [47, 51, 52, 57]. Our team in particular demonstrated that trazodone, a serotonin antagonist and reuptake inhibitor, reduced irritability, agitation, and depressive symptoms in FTD [58]. The much better tolerance profile and apparent efficacy of serotonin-acting drugs logically imposed them as the mainstay of FTD treatment in our network.

\section{Strengths and limitations}

This naturalistic study of a 6-year period is rooted in 23 years of data sharing and harmonization across a regionwide memory clinic network [24]. It allowed to analyze the trends of real-life FTD diagnosis and care over time. We reached a considerable number of new patients per year, equivalent to the one of nation-wide MC networks. Analyzing the characteristics of consecutive FTD patients first referred between 2010 and 2016 allowed us to focus on patients in which the diagnosis was made using the new criteria for bvFTD and lvFTD [3] and 
strengthened by follow-up. By considering a wide spectrum of FTD variants, we included patients that are often withdrawn from FTD cohorts.

Our survey confirmed many previously published data, which reinforces of the quality and validity of our database. We showed that approximately two thirds of FTD patients had a behavioral variant (bvFTD), and 17\% had a language variant, which matches other databases [9, $59,60]$. We, as others, found a sex ratio of approximately 1:1 in FTD [9]. Thirty-five percent of our FTD patients had a family history of neuropsychiatric disease, in agreement with the literature $[14,61]$. Only our rate of mutations was lower than previously reported since a mutation was identified in C9ORF72, MATP, or GRN in only $6 \%$ of the FTD patients that had a genetic analysis, against $10-15 \%$ in the literature [62]. Last but not the least, pathological diagnoses when available matched the clinical diagnoses, confirming the high accuracy of the clinical diagnoses made in a structured regional network and confirmed by a prolonged follow-up.

Our survey has however a few limitations. First, important data are not systematically populated in our database. We still lack accurate cognitive, functional, or disease-specific scales to assess disease progression. Furthermore, the mean follow-up of $\sim 2$ years precludes a comprehensive overview of FTD progression in many of our patients. We also acknowledge a selection bias due to the different networks involved in movement disorders and dementia care in our region, an issue that had been acknowledged in similar studies. Patients with overt motor neuron disease at presentation were not included because they were referred to a specialized regional care pathway rather than to memory clinics. Likewise, the PSP and CBS patients that were referred to our memory clinics were probably the ones presenting early behavioral and/or cognitive changes. Conversely, PSP and CBS with prominent motor symptoms were likely to be followed in movement disorders clinics, where secondary referral to MCs is not systematic. Still, our incidence rate compares to the ones of two regional cohorts including the full FTD spectrum [17, 29].

\section{Conclusion and outlook}

Overall, our study showed that FTD syndromes have specific clinical features, different progression patterns, and therapeutic strategies. Yet, even in a region with an organized memory clinic network, FTD is still overlooked and diagnosis wandering remains longer than in AD. Psychiatric, amnestic, and/or late-onset presentations of FTD are particularly treacherous, and the overlap between cognitive/behavioral and motor presentations leads to an underestimation of the motoric presentations of FTD in memory clinics.
There is an obvious need of accurate FTD biomarkers to improve FTD diagnosis. Until and even after the avenue of such biomarkers, neuropsychology has and will have a role to play at a limited cost. The development of novel tests exploring new domains of social cognition beyond mentalization and emotion recognition is a steppingstone in this direction. Social cognition deficits have been found to be a reliable and effective cognitive marker of FTD, especially in patients with a psychiatric [63] or amnestic $[64,65]$ presentations. Social cognition deficits are probably underestimated in MFTD as well [66], advocating for a more systematic assessment of social cognition in memory, geriatric, movement disorders, and psychiatry clinics. In order to improve FTD diagnosis, the classical boundaries between specialties should be broken. Indeed, it is only through a harmonization in diagnostic procedures and databases involving geriatricians, movement disorders specialists, old-age psychiatrists, neuropsychologists, speech-therapists, and memory clinics that the real scope of FTD will be thoroughly apprehended. The gathering of these different disciplines into consortiums such as the Centers of Excellence in Neurodegeneration (CoEN) responds to this objective.

Additionally, initiatives are needed to raise awareness on FTD in the general population. At the eve of diseasemodifying therapies, misdiagnosis of FTD may already be a loss of opportunity for patients.

\section{Abbreviations}

AD: Alzheimer's disease; AChEls: Acetylcholinesterase inhibitors; ANOVA: Analysis of variance; bvFTD: Behavioral variant of frontotemporal dementia; C9ORF72: Chromosome 9 open reading frame 72; CBD: Corticobasal degeneration; CBS: Corticobasal syndrome; CSF: Cerebrospinal fluid; FTD: Frontotemporal dementia; IADL: Instrumental Activities of Daily Living; MAPT: Microtubule-associated protein tau; MC: Memory Clinic; mFTD : Frontotemporal lobar degeneration with primarily motor symptoms; MMSE : Mini mental state examination; MRRC : Memory Resources and Research Center; IVFTD : Language variants of frontotemporal lobar degeneration; PPA: Primary progressive aphasia; PGRN : Progranuline; PSP: Progressive supranuclear palsy; TDP-43: TAR DNA-binding protein 43

\section{Acknowledgements \\ The authors thank Catherine Ficot, Nathalie Jourdan, and Brigitte Leprince for their continuous energetic contribution to the network as well as the overall Méotis. \\ The authors also thank Antoine Lamer and the MatchID team for their help in retrieving the dates of death.}

\section{Authors' contributions}

ML did the study concept and design, did the analysis and interpretation of data, drafted the manuscript, revised the manuscript for important intellectual content, did the statistical analysis, and contributed administrative support. MB did the study concept and design, did the interpretation of data, and revised the manuscript for important intellectual content. ES did the statistical analysis and revised the manuscript for important intellectual content. EM did the analysis and interpretation of data and revised the manuscript for important intellectual content. CAB, SB, PC, $Y C, V D, F L, M A M, A R S, F P$, and the Méotis network collected the data and revised the manuscript for important intellectual content. MW did the statistical analysis of the incidence and survival. ILB performed the genetic 
analysis. TL did the study concept and design, did the analysis and interpretation of data, drafted the manuscript, revised the manuscript for important intellectual content, and supervised the overall study. The authors read and approved the final manuscript.

\section{Authors' information}

Not applicable

\section{Funding}

The study was partially funded by laboratory of excellence DistAlz and ML PhD was funded by Human Brain Project.

\section{Availability of data and materials}

The datasets considered in the current study are available from the corresponding author on reasonable request.

\section{Ethics approval and consent to participate}

The database was declared to the ad hoc commission (Commission Nationale Informatique et Libertés (CNIL)) protecting personal data (\#2146189 V1). Privacy and confidentiality rules were respected.

\section{Consent for publication}

Not applicable

\section{Competing interests}

The authors declare that they have no competing interests.

\section{Author details}

'Univ. Lille, Inserm, CHU Lille, Lille Neuroscience \& Cognition, CNRMAJ, LiCEND, DistAlz, F-59000 Lille, France. ${ }^{2}$ Biostastitic department, CHU Lille, DistALz, Lille, France. ${ }^{3}$ Univ. Lille, Inserm, CHU Lille, F-59000 Lille, France. ${ }^{4}$ Sorbonne Université, Inserm U1127, CNRS UMR 7225, Institut du Cerveau (ICM), AP-HP - Hôpital Pitié-Salpêtrière, Paris, France. ${ }^{5}$ Centre de référence des démences rares ou précoces, IM2A, Département de Neurologie, AP-HP Hôpital Pitié-Salpêtrière, Paris, France. ${ }^{6}$ Department of Public Health, $\mathrm{CHU}$ Lille, F-59000 Lille, France.

Received: 29 July 2020 Accepted: 16 December 2020

Published online: 08 January 2021

\section{References}

1. Ratnavalli E, Brayne C, Dawson K, Hodges JR. The prevalence of frontotemporal dementia. Neurology. 2002;58:1615-21.

2. Lashley T, Rohrer JD, Mead S, Revesz T. Review: an update on clinical, genetic and pathological aspects of frontotemporal lobar degenerations. Neuropathol Appl Neurobiol. 2015;41:858-81.

3. Rascovsky K, Hodges JR, Knopman D, Mendez MF, Kramer JH, Neuhaus J, et al. Sensitivity of revised diagnostic criteria for the behavioural variant of frontotemporal dementia. Brain. 2011;134:2456-77.

4. Gorno-Tempini ML, Hillis AE, Weintraub S, Kertesz A, Mendez M, Cappa SF, et al. Classification of primary progressive aphasia and its variants. Neurology. 2011;76:1006-14.

5. Höglinger GU, Respondek G, Stamelou M, Kurz C, Josephs KA, Lang AE, et al. Clinical diagnosis of progressive supranuclear palsy: the movement disorder society criteria. Mov Disord. 2017:32:853-64.

6. Josephs KA, Hodges JR, Snowden JS, Mackenzie IR, Neumann M, Mann DM, et al. Neuropathological background of phenotypical variability in frontotemporal dementia. Acta Neuropathol. 2011;122:137-53.

7. Kertesz A, McMonagle P, Blair M, Davidson W, Munoz DG. The evolution and pathology of frontotemporal dementia. Brain. 2005;128:1996-2005.

8. Greaves $C V$, Rohrer JD. An update on genetic frontotemporal dementia. J Neurol. 2019;266:2075-86.

9. Hogan DB, Jetté N, Fiest KM, Roberts JI, Pearson D, Smith EE, et al. The prevalence and incidence of frontotemporal dementia: a systematic review. Can J Neurol Sci. 2016;43(Suppl 1):S96-109.

10. Religa D, Fereshtehnejad S-M, Cermakova P, Edlund A-K, Garcia-Ptacek S, Granqvist N, et al. SveDem, the Swedish Dementia Registry - a tool for improving the quality of diagnostics, treatment and care of dementia patients in clinical practice. Plos One. 2015;10:e0116538.

11. Van der Flier WM, Scheltens P. Amsterdam dementia cohort: performing research to optimize care. J Alzheimer's Dis Jad. 2018;62:1091-111.
12. Barker WW, Luis CA, Kashuba A, Luis M, Harwood DG, Loewenstein D, et al. Relative frequencies of Alzheimer disease, Lewy body, vascular and frontotemporal dementia, and hippocampal sclerosis in the State of Florida Brain Bank. Alz Dis Assoc Dis. 2002;16:203-12.

13. Beach TG, Adler CH, Sue LI, Serrano G, Shill HA, Walker DG, et al. Arizona study of aging and neurodegenerative disorders and brain and body donation program. Neuropathology. 2015;35:354-89.

14. Rosso SM, Kaat LD, Baks T, Joosse M, de Koning I, Pijnenburg Y, et al. Frontotemporal dementia in the Netherlands: patient characteristics and prevalence estimates from a population-based study. Brain. 2003;126:201622.

15. Van de Vliet D, de Vugt ME, Bakker C, Pijnenburg YAL, Vernooij-Dassen MJFJ, Koopmans RTCM, et al. Time to diagnosis in young-onset dementia as compared with late-onset dementia. Psychol Med. 2013;43:423-32.

16. Zhao M, Lv X, Tuerxun M, He J, Luo B, Chen W, et al. Delayed help seeking behavior in dementia care: preliminary findings from the Clinical Pathway for Alzheimer's Disease in China (CPAD) study. Int Psychogeriatr. 2015;28: 211-9.

17. Coyle-Gilchrist ITS, Dick KM, Patterson K, Rodríquez PV, Wehmann E, Wilcox $A$, et al. Prevalence, characteristics, and survival of frontotemporal lobar degeneration syndromes. Neurology. 2016:86:1736-43.

18. Clinical and neuropathological criteria for frontotemporal dementia. The Lund and Manchester Groups. J Neurology Neurosurg Psychiatry. 1994;57: 416. https://pubmed.ncbi.nlm.nih.gov/8163988/.

19. Neary D, Snowden JS, Gustafson L, Passant U, Stuss D, Black S, et al. Frontotemporal lobar degeneration. Neurology. 1998;51:1546-54.

20. Bertoux M, de Souza LC, Corlier F, Lamari F, Bottlaender M, Dubois B, et al. Two distinct amnesic profiles in behavioral variant frontotemporal dementia. Biol Psychiatry. 2014;75:582-8.

21. Ducharme S, Price BH, Larvie M, Dougherty DD, Dickerson BC. Clinical approach to the differential diagnosis between behavioral variant frontotemporal dementia and primary psychiatric disorders. Am J Psychiatry. 2015;172:827-37.

22. Jack CR, Knopman DS, Chételat G, Dickson D, Fagan AM, Frisoni GB, et al. Suspected non-Alzheimer disease pathophysiology--concept and controversy. Nat Rev Neurol. 2016:12:117-24.

23. Winblad B, Amouyel P, Andrieu S, Ballard C, Brayne C, Brodaty H, et al. Defeating Alzheimer's disease and other dementias: a priority for European science and society. Lancet Neurol. 2016;15:455-532.

24. Chen $\mathrm{Y}$, Lebouvier $\mathrm{T}$, Skrobala $\mathrm{E}$, et al. Twenty-year trends in patient referrals throughout the creation and development of a regional memory clinic network. Alzheimer's Dement. 2020;6:e12048.

25. Litvan I, Agid Y, Calne D, Campbell G, Dubois B, Duvoisin RC, et al. Clinical research criteria for the diagnosis of progressive supranuclear palsy (SteeleRichardson-Olszewski syndrome): report of the NINDS-SPSP International Workshop. Neurology. 1996;47:1-9.

26. Armstrong MJ, Litvan I, Lang AE, Bak TH, Bhatia KP, Borroni B, et al. Criteria for the diagnosis of corticobasal degeneration. Neurology. 2013;80:496-503.

27. Folstein MF, Folstein SE, McHugh PR. "Mini-mental state". A practical method for grading the cognitive state of patients for the clinician. J Psychiatr Res. 1975;12:189-98.

28. Pérès K, Helmer C, Amieva H, Orgogozo J-M, Rouch I, Dartigues J-F, et al. Natural history of decline in instrumental activities of daily living performance over the 10 years preceding the clinical diagnosis of dementia: a prospective population-based study. J Am Geriatr Soc. 2008:56:37-44.

29. Logroscino G, Piccininni M, Binetti G, Zecca C, Turrone R, Capozzo R, et al. Incidence of frontotemporal lobar degeneration in Italy: the Salento-Brescia Registry study. Neurology. 2019;92:e2355-63.

30. Gurvit H, Emre M, Tinaz S, Bilgic B, Hanagasi H, Sahin H, et al. The prevalence of dementia in an urban Turkish population. Am J Alzheimer's Dis Other Dementias. 2008;23:67-76.

31. Gascón-Bayarri J, Reñé R, Barrio JLD, Pedro-Cuesta JD, Ramón JM, Manubens JM, et al. Prevalence of dementia subtypes in El Prat de Llobregat, Catalonia, Spain: The PRATICON Study. Neuroepidemiology. 2007;28:224-34

32. Andreasen N, Blennow K, Sjödin C, Winblad B, Svärdsudd K. Prevalence and incidence of clinically diagnosed memory impairments in a geographically defined general population in Sweden. Neuroepidemiology. 1999:18:144-55.

33. Francis PT, Costello H, Hayes GM. Brains for dementia research: evolution in a longitudinal brain donation cohort to maximize current and future value. J Alzheimer's Dis. 2018;66:1635-44. 
34. Seo SW, Thibodeau M-P, Perry DC, Hua A, Sidhu M, Sible I, et al. Early vs late age at onset frontotemporal dementia and frontotemporal lobar degeneration. Neurology. 2018;90:e1047-56.

35. Borroni B, Agosti C, Bellelli G, Padovani A. Is early-onset clinically different from late-onset frontotemporal dementia? Eur J Neurol. 2008;15:1412-5.

36. Nilsson C, Waldö ML, Nilsson K, Santillo A, Vestberg S. Age-related incidence and family history in frontotemporal dementia: data from the Swedish Dementia Registry. Plos One. 2014;9:e94901.

37. Turcano $P$, et al. Incidence of frontotemporal disorders in Olmsted County: a population-based study. Alzheimer's Dementia. 2020;16:482-90.

38. Ducharme $S$, et al. Recommendations to distinguish behavioural variant frontotemporal dementia from psychiatric disorders. Brain. 2020;143:1632-50.

39. Pasquier F, Richard F, Lebert F. Natural history of frontotemporal dementia: comparison with Alzheimer's disease. Dement Geriatr Cogn Disord. 2004;17: 253-7.

40. Woolley JD, Khan BK, Murthy NK, Miller BL, Rankin KP. The diagnostic challenge of psychiatric symptoms in neurodegenerative disease: rates of and risk factors for prior psychiatric diagnosis in patients with early neurodegenerative disease. J Clin Psychiatry. 2011;72:126-33.

41. Besser LM, Teylan MA, Nelson PT. Limbic-predominant age-related TDP-43 encephalopathy (LATE): Clinical and Neuropathological. J Neuropathol Exp Neurol. Brain. 2020;79:305-13.

42. Bertoux $M$, et al. Does amnesia specifically predict Alzheimer's pathology? A neuropathological study. Neurobiol Aging. 2020;95:123-30.

43. Ulugut Erkoyun $\mathrm{H}$, et al. A clinical-radiological framework of the right temporal variant of frontotemporal dementia. Brain J Neurol. 2020;143: 2831-43.

44. Mioshi E, Hodges JR. Rate of change of functional abilities in frontotemporal dementia. Dement Geriatr Cogn. 2009;28:419-26.

45. Larner AJ, Hancock P. Re: activities of daily living in frontotemporal dementia and Alzheimer disease. Neurology. 2008;70:658.

46. Tan KS, Libon DJ, Rascovsky K, Grossman M, Xie SX. Differential longitudinal decline on the Mini-Mental State Examination in frontotemporal lobar degeneration and Alzheimer disease. Alzheimer Dis Assoc Disord. 2013;27: 310-5.

47. Trieu C, Gossink F, Stek ML, Scheltens P, Pijnenburg YAL, Dols A Effectiveness of pharmacological interventions for symptoms of behaviora variant frontotemporal dementia: a systematic review. Cogn Behav Neurol. 2020;33:1-15.

48. Hu B, Ross L, Neuhaus J, Knopman D, Kramer J, Boeve B, et al. Off-label medication use in frontotemporal dementia. Am J Alzheimer's Dis Other Dement. 2010;25:128-33.

49. Mendez MF, Shapira JS, McMurtray A, Licht E. Preliminary findings: behavioral worsening on donepezil in patients with frontotemporal dementia. Am J Geriatric Psychiatry. 2012;15:84-7.

50. Vercelletto $M$, Boutoleau-Bretonnière $C$, Volteau C, Puel M, Auriacombe S, Sarazin $M$, et al. Memantine in behavioral variant frontotemporal dementia: negative results. J Alzheimer's Dis Jad. 2011;23:749-59.

51. Tsai RM, Boxer AL. Treatment of frontotemporal dementia. Curr Treat Options Neurol. 2014;16:841-15.

52. Tsai RM, Boxer AL. Therapy and clinical trials in frontotemporal dementia: past, present, and future. J Neurochem. 2016;138(Suppl 1):211-21.

53. Ringman JM, Schneider L. Treatment options for agitation in dementia. Curr Treat Option Neurol. 2019;21:30.

54. Kerssens CJ, Kerrsens CJ, Pijnenburg YAL. Vulnerability to neuroleptic side effects in frontotemporal dementia. Eur J Neurol. 2008;15:111-2.

55. Pijnenburg YAL, Sampson EL, Harvey RJ, Fox NC, Rossor MN. Vulnerability to neuroleptic side effects in frontotemporal lobar degeneration. Int J Geriatr Psych. 2002;18:67-72.

56. Gill SS, Anderson GM, Fischer HD, Bell CM, Li P, Normand S-LT, et al. Syncope and its consequences in patients with dementia receiving cholinesterase inhibitors: a population-based cohort study. Arch Intern Med. 2009;169:867-73.

57. Huey ED, Putnam KT, Grafman J. A systematic review of neurotransmitter deficits and treatments in frontotemporal dementia. Neurology. 2006;66:17-22.

58. Lebert F, Stekke W, Hasenbroekx C, Pasquier F. Frontotemporal dementia: a randomised, controlled trial with trazodone. Dement Geriatr Cogn Disord. 2004;17:355-9.

59. Johnson JK, Diehl J, Mendez MF, Neuhaus J, Shapira JS, Forman M, et al. Frontotemporal lobar degeneration: demographic characteristics of 353 patients. Arch Neurol. 2005;62:925-30.
60. Ioannidis $P$, Konstantinopoulou E, Maiovis P, Karacostas D. The frontotemporal dementias in a tertiary referral center: classification and demographic characteristics in a series of 232 cases. J Neurol Sci. 2012;318: $171-3$.

61. Goldman JS, Farmer JM, Wood EM, Johnson JK, Boxer A, Neuhaus J, et al. Comparison of family histories in FTLD subtypes and related tauopathies. Neurology. 2005;65:1817-9.

62. Sellami L, Saracino D, Ber IL. Genetic forms of frontotemporal lobar degeneration: current diagnostic approach and new directions in therapeutic strategies. Rev Neurol. (Paris) 2020;176:571-81.

63. Bertoux M, Delavest M, Souza LC de, Funkiewiez A, Lépine J-P, Fossati $P$, et al. Social cognition and emotional assessment differentiates frontotemporal dementia from depression. J Neurology Neurosurg Psychiatry 2012;83:411

64. Bertoux M, Funkiewiez A, O'Callaghan C, Dubois B, Hornberger M. Sensitivity and specificity of ventromedial prefrontal cortex tests in behavioral variant frontotemporal dementia. Alzheimer's Dement. 2013;9:S84-94.

65. Bertoux M, Ramanan S, Slachevsky A, Wong S, Henriquez F, Musa G, et al. So close yet so far: executive contribution to memory processing in behavioral variant frontotemporal dementia. J Alzheimers Dis. 2016;54:100514.

66. Ghosh BCP, Calder AJ, Peers PV, Lawrence AD, Acosta-Cabronero J, Pereira $J M$, et al. Social cognitive deficits and their neural correlates in progressive supranuclear palsy. Brain. 2012;135:2089-102.

\section{Publisher's Note}

Springer Nature remains neutral with regard to jurisdictional claims in published maps and institutional affiliations.
Ready to submit your research? Choose BMC and benefit from:

- fast, convenient online submission

- thorough peer review by experienced researchers in your field

- rapid publication on acceptance

- support for research data, including large and complex data types

- gold Open Access which fosters wider collaboration and increased citations

- maximum visibility for your research: over $100 \mathrm{M}$ website views per year

At $\mathrm{BMC}$, research is always in progress.

Learn more biomedcentral.com/submissions 